\title{
Drosophila melanogaster as a model for lead neurotoxicology and toxicogenomics research
}

\section{Helmut V. B. Hirsch ${ }^{1}$, Gregory Lnenicka ${ }^{1}$, Debra Possidente ${ }^{2}$, Bernard Possidente $^{2}$, Mark D. Garfinkel $^{3}$, Luan Wang ${ }^{4}$, Xiangyi Lu ${ }^{4}$ and Douglas M. Ruden ${ }^{4,5 *}$}

\author{
' Department of Biological Sciences, University at Albany, State University of New York, Albany, NY, USA \\ 2 Department of Biology, Skidmore College, Saratoga Springs, NY, USA \\ ${ }^{3}$ Department of Environmental Health Sciences, University of Alabama at Birmingham, Birmingham, AL, USA \\ ${ }^{4}$ Institute of Environmental Health Sciences, Wayne State University, Detroit, MI, USA \\ ${ }^{5}$ Department of Obstetrics and Gynecology, C. S. Mott Center for Human Growth and Development, Wayne State University, Detroit, MI, USA
}

\section{Edited by:}

Michael Aschner, Vanderbilt University

Medical Center, USA

Reviewed by:

Eugenia Xu, Raymond and Beverly

Sackler Foundation, USA

Marcos De Donato, Cornell

University, USA

${ }^{*}$ Correspondence:

Douglas M. Ruden, Institute of

Environmental Health Sciences,

Wayne State University, Detroit,

MI 48201, USA. e-mail:

douglasr@wayne.edu
Drosophila melanogaster is an excellent model animal for studying the neurotoxicology of lead. It has been known since ancient Roman times that long-term exposure to low levels of lead results in behavioral abnormalities, such as what is now known as attention deficit hyperactivity disorder (ADHD). Because lead alters mechanisms that underlie developmental neuronal plasticity, chronic exposure of children, even at blood lead levels below the current CDC community action level $(10 \mu \mathrm{g} / \mathrm{dl})$, can result in reduced cognitive ability, increased likelihood of delinquency, behaviors associated with ADHD, changes in activity level, altered sensory function, delayed onset of sexual maturity in girls, and changes in immune function. In order to better understand how lead affects neuronal plasticity, we will describe recent findings from a Drosophila behavioral genetics laboratory, a Drosophila neurophysiology laboratory, and a Drosophila quantitative genetics laboratory who have joined forces to study the effects of lead on the Drosophila nervous system. Studying the effects of lead on Drosophila nervous system development will give us a better understanding of the mechanisms of $\mathrm{Pb}$ neurotoxicity in the developing human nervous system.

\section{Keywords: Drosophila, toxicology, toxicogenomics, behavioral toxicology}

\section{PART 1: OTL MAPPING: BEHAVIORS AND TOXINS BEHAVIOR AS A OTL ENDPOINT FOR TOXICOLOGY STUDIES}

There are two main reasons why behavioral assays are so useful in detecting effects of toxins. First is their richness. Behavior can be described as an ongoing, generally complex, spatio-temporal pattern; subtle changes in that pattern can signal the action of verylow doses of a toxin well before there are dramatic changes in organ systems: in children overt clinical encephalopathy is associated with blood lead levels of $80-100 \mu \mathrm{g} / \mathrm{dl}$, while changes in intelligence quotient (IQ) and learning occur at or below a tenth of that dose (UNEP, 2010). Second is that even subtle toxin-dependent changes in behavior can have consequences for well-being which makes them meaningful and relevant.

Since chronic developmental exposure to toxins affects assembly of the neuronal and hormonal systems mediating adult behavior, their effects are generally more pronounced than that following acute adult exposure. Developmental exposure to sub-lethal and sub-teratogenic levels of toxins may alter or degrade physiological and behavioral mechanisms in a quantitative manner. Examples include effects of low doses of lead on behavior in fruit flies (Hirsch et al., 2003), cognitive effects of polychlorobiphenyls in people (Faroon et al., 2000), lead effects on cognitive function in people (Counter et al., 1998), and the effects of aluminum on adult behavior and developmental rate of their offspring in mice (Abu-Taweel et al., 2012). Once toxin-dependent behavioral changes are observed, the next steps may include study of their underlying physiological effects, for example, effects of lead on synaptic function in fruit fly larvae $(\mathrm{He}$ et al., 2009).

The bulk of what is known about the effects of various toxins is based on studies focusing on a single toxin; combinations of toxins can have additive, protective, or synergistic effects (Rai et al., 2010; Singh et al., 2010).

\section{DROSOPHILA BEHAVIORAL OTLS}

Quantitative trait locus (QTL) mapping is used for unbiased genome-wide screens to identify genetic loci causing variation in a trait, but they are most efficient when large numbers of individual genomes can be assayed. The large sample sizes required for precise QTL analysis favors model organisms that can be bred in the lab easily and inexpensively, and traits that are simple to assay yet rich in their information content and thus provide sensitive measures of effects of toxins are especially desirable.

Drosophila is an ideal model organism for both efficiency and genetic analysis, since they are easy and relatively inexpensive to breed and maintain in large numbers, and have a surprising degree of genetic homology to mammals (Mackay and Anholt, 2006). Because of the small physical size of Drosophila many phenotypic traits can be difficult to assay rapidly or quantitatively. Exceptions include very simple traits such as bristle number, or 
those easy to quantify such as number of offspring; others may be easy to automate given an investment in the required technical support.

Automated phenotypic assays in Drosophila tend to favor either gene expression during embryogenesis, where molecular marker extraction and detection can be automated, or locomotor behavior which, by using Drosophila Activity Monitors (DAMs), can be recorded relatively easily for large numbers of flies over periods of many days (Figure 1A; TriKinetics Inc., Waltham, MA, USA; Rosato and Kyriacou, 2006; Rosato et al., 2006).

The richness of the data gathered using DAMs makes it an ideal automated assay for genetic analysis of toxins. DAM data always provides, at a minimum, an estimate of mean locomotor activity level for the duration of the experiment. However, by measuring locomotor activity under the influence of a controlled light-dark cycle the photosensitivity pattern of activity can be analyzed. Using regulated light-dark cycles allows for determining the distribution of activity between the two phases of the photoperiod; the effect of light-dark cycle length other than $24 \mathrm{~h}$; the amplitude of "daily" activity rhythms, the percentage of flies that show significant daily rhythms; and the timing, length, and distribution of sleep periods (Shaw et al., 2000).

Finally, by replacing light-dark cycles with constant darkness the free-running circadian period can be estimated. This provides a direct measure of the output of the biological circadian pacemaker driving the activity rhythm. Toxins that alter the circadian periodicity pattern of locomotor activity are likely, therefore, to perturb the circadian synchronization of many different biological functions regulated by a common central circadian clock. Temporal analysis of behavioral effects of toxins could also suggest the presence of daily rhythms in sensitivity to specific toxins, or in the manifestation of toxic effects. In humans, certain forms of cancer chemotherapy are more effective when administered at specific times of day (Levi et al., 2010; Li et al., 2010); other toxins may also vary in their effects during the rhythm of daytime sunlight and nighttime darkness.

In short, DAMs allow for analysis of toxic effects on both the level of locomotor activity per se and on its temporal pattern of expression (von Mayersbach, 1975; Mayersbach, 1976).

\section{DROSOPHILA ACTIVITY MONITORS AND ACTIVITY ASSAYS}

Behavioral toxicogenetic analysis is a relatively new application of DAM technology. DAMs were originally devised for genetic analysis of the circadian oscillator controlling locomotor activity. Locomotor activity in fruit flies, similar to running wheel activity in rodents, is an excellent reporter phenotype for underlying circadian clock function (Takahashi et al., 2008). Since precise analysis of circadian rhythms require frequent, continuous long-term data sampling for time-series estimation of periodic oscillations in the $24-\mathrm{h}$ range, DAMs are designed to produce a data-rich profile of the locomotor activity of individual fruit flies. Flies are housed, usually singly, in a $5 \mathrm{~mm} \times 60 \mathrm{~mm}$ transparent tube with enough food to last for approximately 2 weeks. A single monitor holds 32 tubes, and a photobeam in the center of each tube tracks locomotor activity as numbers of beam-crossing per unit of time, usually 10-min intervals.

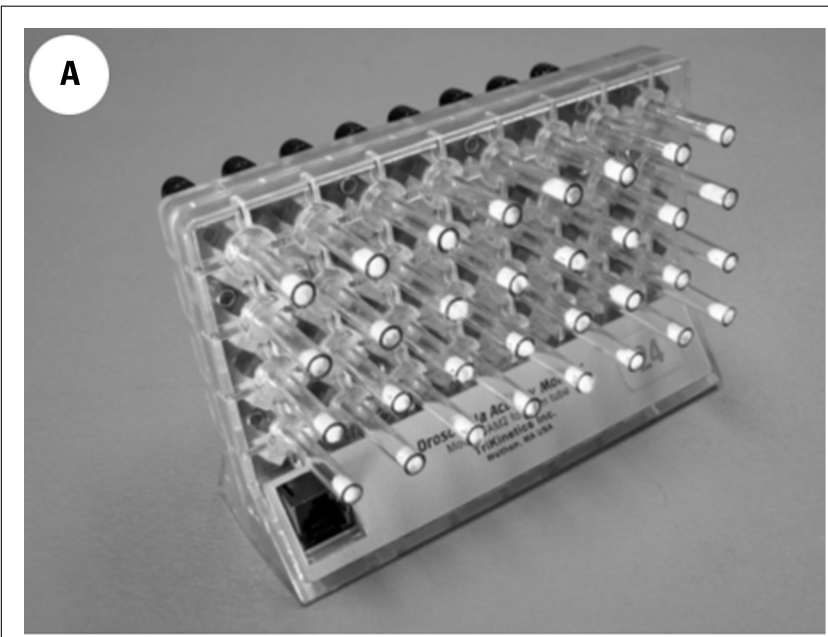

B

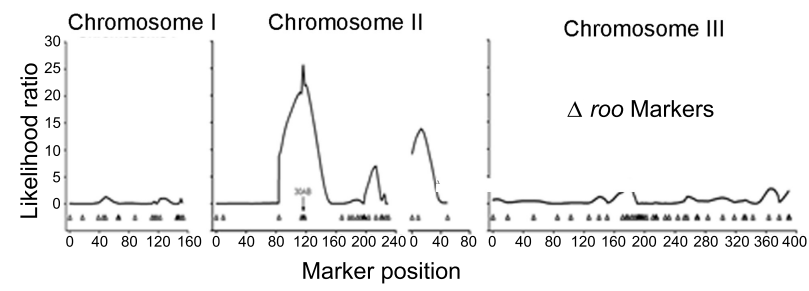

FIGURE 1 | (A) Drosophila Activity Monitor with glass tubes containing one adult fly, food at the far end and a cotton plug visible on the other end of each tube. (B) A behavioral QTL at region 30AB of Drosophila melanogaster chromosome two, for changes in activity level induced by developmental exposure to lead.

Drosophila Activity Monitors permit a variety of experimental designs. It is relatively easy to assay developmental effects of toxins by adding them to the fly culture food during egg, larval, or pupal development, and then monitoring adults. Effects of toxins can also be assayed acutely by adding them to the food in the activity monitor tubes. A single DAM, approximately $5^{\prime \prime}$ long, $4^{\prime \prime}$ high, and $3^{\prime \prime}$ deep holds 32 flies so one refrigeratorsized incubator can house thousands of flies per experiment. The efficiency of DAMs facilitates experimental analysis of sex differences, dose-response curves, sensitive periods during development, interaction among multiple toxins, trans-generational effects, and various types of genetic analysis including behavioral QTL. It is also just as easy to monitor survival time of individual flies to the minute in assays of lethal toxic effects and degrees of resistance to them.

\section{BEHAVIORAL OTL ANALYSIS OF DEVELOPMENTAL EXPOSURE TO TOXINS}

We have identified a toxin-induced behavioral QTL by assaying locomotor activity levels for a set of recombinant inbred (RI) fly lines raised on medium containing $250 \mu \mathrm{M}$ lead acetate or control medium made with $250 \mu \mathrm{M}$ sodium acetate. The variation among the RI fly lines in the difference in mean activity level per line between the two treatments indicated a behavioral QTL in the 
$30 \mathrm{AB}$ region of the second Drosophila chromosome (Figure 1B; Hirsch et al., 2009). This QTL was independently identified as the site of an expression QTL (eQTL) in response to the same lead treatment in the same set of RI lines (RILs). Using the gene expression assay, we further showed the $30 \mathrm{AB}$ eQTL to function as a master modulatory regulator of approximately 70 additional eQTLs throughout the genome in response to developmental lead exposure (Ruden et al., 2009). Current research underway in our labs will map these behavioral QTLs in response to developmental lead exposure more precisely.

\section{PART 2: SYNAPSES AND $\mathrm{Ca}^{2+}$ REGULATION IN DROSOPHILA}

Early findings suggested that chronic $\mathrm{Pb}^{2+}$ exposure could produce its behavioral effects by altering synaptic development. Mammalian studies reported a variety of alterations in synaptic morphology and physiology in the brains of animals exposed to $\mathrm{Pb}^{2+}$ during development (Petit and LeBoutillier, 1979; Kiraly and Jones, 1982; Altmann et al., 1993). Today, the synapse remains a focus for studying the effects of toxins on brain development. Here the Drosophila larval neuromuscular junction (NMJ) offers a distinct advantage since one can compare the same, identified synapse in controls and treated animals (Figure 2). Note that this neuromuscular system is unique even amongst invertebrates because there is a stereotypic pattern of neuromuscular connections where both the presynaptic (motor neuron) and postsynaptic cells (muscle fiber) can be uniquely identified (Keshishian et al., 1996). These neuromuscular synapses have been used to demonstrate the effects of second messengers, celladhesion molecules and their modulators, and impulse activity on synaptic development (Budnik, 1996; Budnik et al., 1996; Davis et al., 1996).

We found that indeed one could detect synaptic abnormalities resulting from chronic $\mathrm{Pb}^{2+}$ exposure at the Drosophila larval NMJ. Initial studies found that the motor terminal formed by motoneuron RP3 on Muscle Fiber 6 (MF6) showed greater variability in size for animals raised in media containing $\mathrm{Pb}^{2+}$ compared to controls (Morley et al., 2003). One could imagine that increasing the variability of synaptic size could result in a loss of fine-tuning of synaptic function; this effect, if seen in the brain, would likely be detrimental to circuit dynamics and behavior. It is noteworthy that this synaptic change was subtle and it likely would have been difficult to detect when making comparisons among a large population of synapses in the mammalian brain. Given that the behavioral effects of toxins, such as $\mathrm{Pb}^{2+}$, can be subtle, it seems reasonable that the effects on synapses might also be small.

$\mathrm{Pb}^{2+}$ affects proteins that bind $\mathrm{Ca}^{2+}$ including those that regulate the intracellular $\mathrm{Ca}^{2+}$ concentration $\left(\left[\mathrm{Ca}^{2+}\right]_{\mathrm{i}}\right)$, such as $\mathrm{Ca}^{2+}$ channels and $\mathrm{Ca}^{2+}$ pumps. Changes in $\mathrm{Ca}^{2+}$ regulation could be particularly important for the synapse since $\left[\mathrm{Ca}^{2+}\right]_{\mathrm{i}}$ controls multiple steps in synaptic development; e.g., growth cone guidance (Jin et al., 2005), synapse formation (Xu et al., 2009), and synapse elimination and stabilization (Pratt et al., 2003; Lohmann and Bonhoeffer, 2008). In addition, altered $\mathrm{Ca}^{2+}$ regulation could influence transmitter release (Zucker, 1996) and both long-term and short-term forms of synaptic plasticity at the mature synapse (Zucker and Regehr, 2002; MacDonald et al., 2006).

Acute $\mathrm{Pb}^{2+}$ exposure blocks $\mathrm{Ca}^{2+}$ channels. This has been demonstrated for a variety of voltage-dependent $\mathrm{Ca}^{2+}$ channels in both invertebrates (Audesirk and Audesirk, 1989) and mammals (Evans et al., 1991). The acute application of micromolar concentrations of $\mathrm{Pb}^{2+}$ can reduce the activity of the plasma membrane $\mathrm{Ca}^{2+}$ ATPase (PMCA) in humans and rats (Mas-Oliva, 1989; Sandhir and Gill, 1994a,b); however the PMCA can be stimulated by low $\mathrm{Pb}^{2+}$ concentrations (Mas-Oliva, 1989; Campagna et al., 2000). In addition, chronic in vivo $\mathrm{Pb}^{2+}$ exposure has been found to produce a persistent inhibition of PMCA activity in human erythrocytes (Campagna et al., 2000) and rat synaptosomes (Sandhir and Gill, 1994a,b).
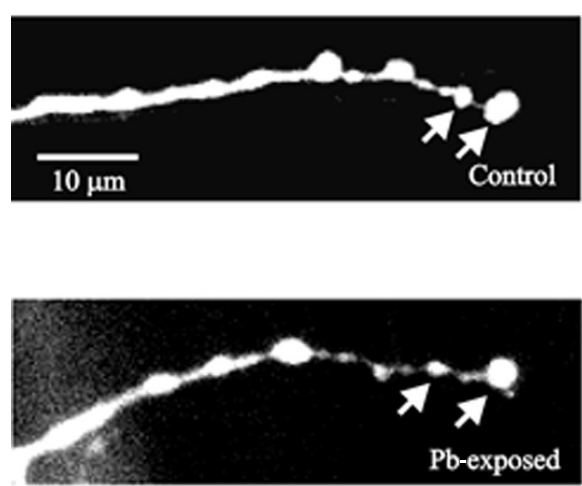

FIGURE 2 | $\mathrm{Ca}^{2+}$ transients recorded from identified synaptic terminals in control and $\mathbf{P b}^{\mathbf{2}+}$-exposed animals. (Left) The RP3 motor terminals on MF6 were filled with OGB-1 in control larvae and in those exposed to $\mathrm{Pb}^{2}+$. The arrows point to two typical synaptic boutons where $\mathrm{Ca}^{2}+$ transients were measured. (Right) Typical $\mathrm{Ca}^{2}+$ transients produced by single action potentials (AP) and AP trains for control and
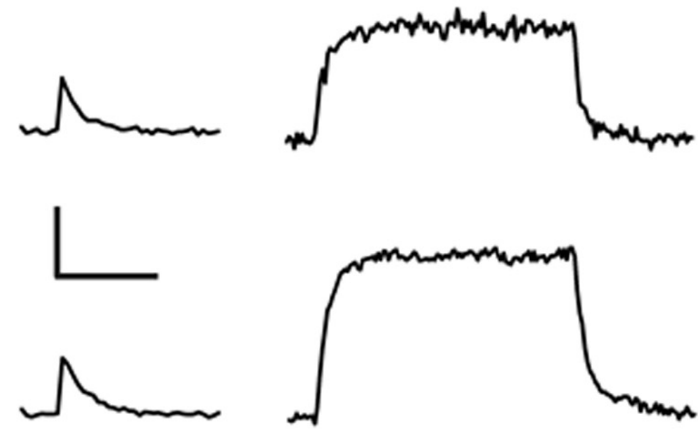

Single AP

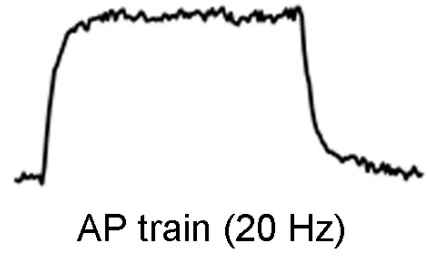

$\mathrm{Pb}^{2+}$-exposed boutons. For single APs, the $\mathrm{Ca}^{2+}$ transients were similar for control and $\mathrm{Pb}^{2+}$-exposed synaptic boutons; however, the $\mathrm{Ca}^{2}+$ transients produced by AP trains were larger and decayed more slowly in $\mathrm{Pb}^{2+}$-exposed boutons compared to controls. Calibration: single $A p-20 \% \Delta F / F, 0.4$ s; AP train $-20 \% \Delta F / F, 2$ s. Adapted from He etal. (2009). 
We studied the effect of chronic $\mathrm{Pb}^{2+}$ exposure on presynaptic $\mathrm{Ca}^{2+}$ regulation at the RP3-to-MF6 synapse in Drosophila larvae (He et al., 2009). $\mathrm{Ca}^{2+}$ indicators were loaded in these motor terminals and we measured the changes in $\left[\mathrm{Ca}^{2+}\right]_{i}$ produced by single action potentials and action potential trains (Figure 2 ). We found that chronic exposure to $\mathrm{Pb}^{2+}$ resulted in a greater increase in $\left[\mathrm{Ca}^{2+}\right]_{i}$ during trains of action potentials and a slower decay of postsynaptic $\left[\mathrm{Ca}^{2+}\right]_{i}$ at the end of the train. This is likely due to a decrease in the activity of the PMCA and it provides an interesting parallel to the effect of $\mathrm{Pb}^{2+}$ exposure on the PMCA in mammals. The direct effect of this large increase in $[\mathrm{Ca}]_{i}$ was that the $\mathrm{Pb}^{2+}$-exposed animals showed greater synaptic facilitation. This was consistent with the residual $\mathrm{Ca}^{2+}$ model for synaptic facilitation (Zucker and Regehr, 2002) and with mammalian studies showing that knocking down expression of the PMCA resulted in enhanced synaptic facilitation (Empson et al., 2007). In that study, reduced PMCA expression also produced changes in neuronal structure (Empson et al., 2007) and it may be that the $\mathrm{Pb}^{2+}$-induced changes in the structure of the larval NMJ resulted from reduced PMCA activity and altered $\mathrm{Ca}^{2+}$ regulation.

In summary, identified NMJs in Drosophila larvae allow for the detection of subtle changes in synaptic structure and function resulting from developmental exposure to toxins. Our evidence suggests that chronic $\mathrm{Pb}^{2+}$ exposure produces parallel synaptic changes in Drosophila and mammals; this would not be surprising given that chemical synapses found in vertebrates and invertebrates are very similar.

\section{PART 3: GENETICAL GENOMICS STUDIES IN DROSOPHILA}

Developmental neurotoxicology research requires an approach that reduces the candidate toxin-regulated genes to a manageable number. Fortunately, a new multi-dimensional strategy has been developed, called genetical genomics, which identifies master modulatory loci that regulate the expression of hundreds of other genes (Jansen and Nap, 2001; Broman, 2005; de Koning and Haley, 2005; Li and Burmeister, 2005; Rockman and Kruglyak, 2006). Genetical genomics combines two methodologies, microarraybased whole transcriptome analyses and extracting QTLs by using RILs. Global gene expression levels are determined for each RIL, and then QTL mapping software (e.g., R/QTL; Broman et al., 2003) is used to find above-threshold statistical associations between specific chromosomal loci and transcript levels for all the genes measured on the microarrays. With QTL analysis, we can identify the specific chromosomal loci that regulate genes, which are referred to as eQTLs (Mueller et al., 2006; West et al., 2007; Majewski and Pastinen, 2011; Zhang et al., 2012).

Our research is the first that combines genetical genomics with toxicogenomics, an experimental approach we call "genetical toxicogenomics" (Ruden et al., 2009). Li et al. (2008) showed that adding environmental perturbations in genetical genomics studies, as we did in our study (Ruden etal., 2009), allows toxin-response genes to be identified. We believe that this genetical toxicogenomics approach will drive the field of toxicology and aid in understanding the effects of toxins such as lead.
Expression QTL analyses were performed for all 18,000 genes and other microarray features by treating the expression level of each gene as a quantitative trait. The genetics of gene expression in RILs can be mapped as eQTLs. Flies from each of 75 RILs were fed, from egg to adult, either control food or lead-treated food (made with $250 \mu \mathrm{M}$ lead acetate). RNA expression analyses of whole adult male flies (5-10 days old) were performed with Affymetrix Dros2 whole genome arrays (18,952 probe sets). Loci that are linked to a gene are called locally acting or ciseQTL, whereas loci that are distantly acting are called trans-eQTLs (Broman, 2005).

The first genetical genomics study to identify genes with significant GxE interactions was done in Caenorhabditis elegans; the authors identified a group of genes with trans-eQTL that are induced by heat shock, which they called plastic QTL (Li et al., 2006). Smith and Kruglyak (2008) recently performed a detailed analysis of GxE-eQTL in yeast (which they call "gxeQTL") grown in either glucose or ethanol as the sole carbon source. Others have identified $\mathrm{GxE}$ interactions in which the environment is a different tissue (e.g., brain vs. liver; Hovatta et al., 2007). In our studies, among the 1,389 genes with cis-eQTL, there were 405 genes unique to control flies and 544 genes unique to lead-treated ones (440 genes had the same cis-eQTLs in both samples).

There were 2,396 genes with trans-eQTL that mapped to 12 major hotspots that met statistical significance $(p<0.05$, chisquared test, based on permutation analyses, on 5-cM windows). Unexpectedly, we identified two hotspots, one located on the second chromosome (polytene region $30 \mathrm{AB}$ ) and one on the third chromosome (polytene region 73D), which co-regulate 33 genes, all of which are induced by lead (Figure 3). We have shown by QTL analysis that marker locus $30 \mathrm{AB}$ contributes to lead-dependent changes in locomotion, which suggests that the genes in the $30 \mathrm{AB}$ hotspot can be used as a functional test to identify both the leaddependent trans-regulatory factor and the common cis-regulatory motifs (Hirsch et al., 2009). We propose that a trans-regulator located at 73D increases expression of its target genes when it binds lead, regardless of the genotype of the 73D hotspot. In contrast, a second trans-regulator located at $30 \mathrm{AB}$ increases expression of the co-regulated target genes only when it has the ORE genotype (Figure 3).

This model can explain the data, but other explanations are possible. For example, the putative trans-regulator could increase the stability of the mRNA of the target genes, or there could be an indirect effect on steady-state mRNA levels. MEME (Multiple Em for Motif Elicitation) analyses of the genes regulated by the $30 \mathrm{AB}$ and $73 \mathrm{D}$ trans-regulators identified both conserved proximal promoter and $3^{\prime}$-untranslated region (UTR) sequences (data not shown). Fine-mapping of the genes that underlie the QTLs and molecular and biochemical analyses should enable us to determine the mechanism involved.

\section{FUTURE STUDIES IN DROSOPHILA NEUROTOXICOLOGY}

In this review, we describe recent results from several laboratories that are collaborating on studying the effects of lead on the developing Drosophila nervous system. The Possidente and Hirsch 


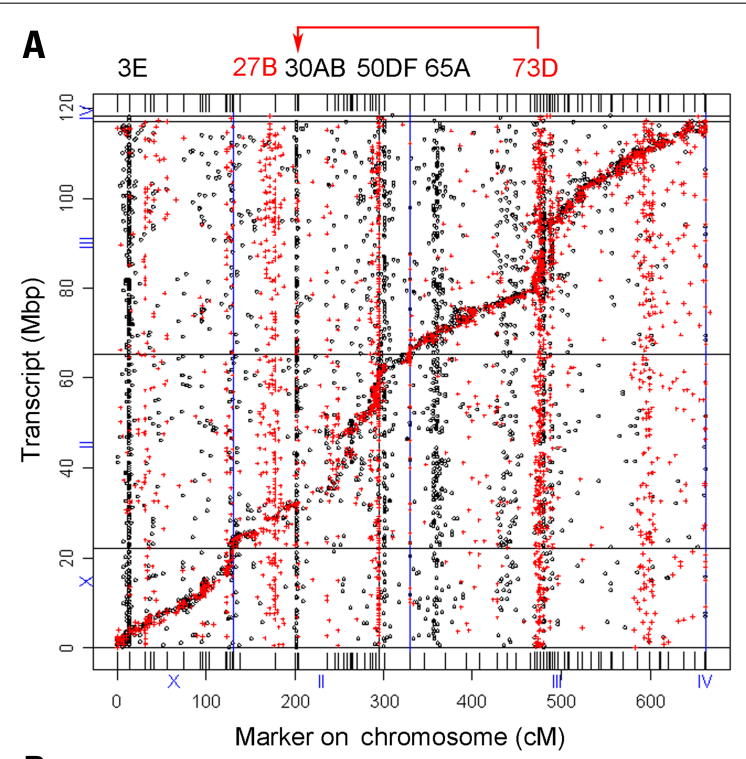

B

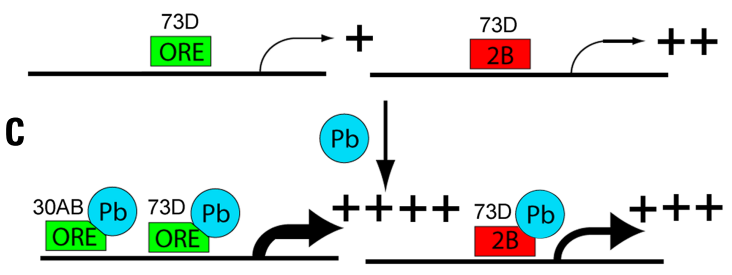

FIGURE 3 | Genetical genomics results of Drosophila recombinant inbred lines with developmental lead exposure. (A) The $x$-axis is the chromosome locations of the cis- and trans-eQTLs in centimorgans (cM). The $y$-axis is the location of the transcript in mega-base pairs (Mbp). The eQTLs in control (red) and the eQTLs treated (black) are indicated as dots and crosses, respectively. The locations of the control trans-eQTL are shown on top in red and the lead-treated trans-eQTL locations are shown in black letters. Many of the eQTL at 70D in the control flies shift to 30AB in the lead-treated flies (red arrow at top). (B) The trans-activator encoded by the 73D hotspot is required for basal transcription of the 33 genes co-regulated by both $73 \mathrm{D}$ and $30 \mathrm{AB}$ master modulatory genes. (C) In the presence of lead, the trans-regulator encoded by the 73D hotspot further increases steady-state mRNA levels when it has either the ORE or 2B genotype. However, the trans-regulator encoded by the $30 \mathrm{AB}$ hotspot further increases steady-state mRNA levels for the 33 target genes only when it has the ORE genotype. Adapted from Ruden et al. (2009).

\section{REFERENCES}

Abu-Taweel, G. M., Ajarem, J. S., and Ahmad, M. (2012). Neurobehavioral toxic effects of perinatal oral exposure to aluminum on the developmental motor reflexes, learning, memory and brain neurotransmitters of mice offspring. Pharmacol. Biochem. Behav. 101, 49-56.

Altmann, L., Weinsberg, F., Sveinsson, K., Lilienthal, H., Wiegand, H., and Winneke, G. (1993). Impairment of long-term potentiation and learning following chronic lead exposure. Toxicol. Lett. 66, 105-112.

Audesirk, G., and Audesirk, T. (1989). Effects of in vitro lead exposure on voltage-sensitive calcium channels differ among cell types in central neurons of Lymnaea stagnalis. Neurotoxicology 10 , 659-669.

Broman, K. W. (2005). Mapping expression in randomized rodent genomes. Nat. Genet. 37, 209-210. [see comments].

Broman, K. W., Wu, H., Sen, S., and Churchill, G. A. (2003). R/qtl: QTL mapping in experimental crosses. Bioinformatics 19, 889-890.

Budnik, V. (1996). Synapse maturation and structural plasticity at Drosophila neuromuscular junctions. Curr. Opin. Neurobiol. 6, 858-867.

laboratories study the effects of lead on behavioral quantitative traits. The Lnenicka laboratory studies the effects of lead on the NMJ in the larvae. The Ruden, Lu, and Garfinkel laboratories study the effects of lead on gene expression using quantitative genetics techniques.

Further innovations to the field of genetical genomics will make use of next-generation RNA sequencing (RNA-seq) rather than gene expression microarrays. RNA-seq will allow us not only to accurately determine gene expression levels, but also to analyze alternative splicing products of genes, thereby adding a new dimension to these studies. Since over $90 \%$ of human genes and a similar percentage of Drosophila genes are alternatively spliced, RNA-seq could provide some exciting and novel findings about environmental regulation of alternative mRNA splicing. The potential for RNA-seq to supplant microarrays requires, however, the development of robust statistical tools for analyzing the resulting huge datasets.

Adding metabolomics profiling to these studies will allow us to identify metabolite QTL (mQTL) that are specific for lead. To our knowledge mQTL analyses have never been conducted in any organism, although genome-wide association studies combined with metabolic profiling were conducted in a recent human study (Prakash, 2011; Suhre et al., 2011). The human study identified 37 genetic loci associated with blood metabolite concentrations, of which 25 showed effect sizes of $10-60 \%$ which is extraordinarily high for genome-wide association studies (Prakash, 2011; Suhre et al., 2011).

We are now entering the stage of understanding the complex genetic pathways that are affected by developmental lead exposure. The sophisticated genetic analyses that are possible in Drosophila will soon allow us to manipulate these pathways to better understand how they are affected by lead. The sequencing of several Drosophila strains and studying the effects of lead on neurodevelopment in these strains will allow us to better understand the evolution of lead-sensitive pathways. Together, these studies will provide a better understanding of the health effects of lead in humans.

\section{ACKNOWLEDGMENTS}

This work was supported by NIH R01 grants ES012933 to Douglas M. Ruden, and DK071073 to Xiangyi Lu.

Budnik, V., Koh, Y. H., Guan, B., Hartmann, B., Hough, C., Woods, D., and Gorczyca, M. (1996). Regulation of synapse structure and function by the Drosophila tumor suppressor gene dlg. Neuron 17, 627-640.

Campagna, D., Huel, G., Hellier, G., Girard, F., Sahuquillo, J., FagotCampagna, A., Godin, J., and Blot, P. (2000). Negative relationships between erythrocyte Ca-pump activity and lead levels in mothers and newborns. Life Sci. 68, 203-215.

Counter, S. A., Buchanan, L. H., Rosas, H. D., and Ortega, F. (1998).
Neurocognitive effects of chronic lead intoxication in Andean children. J. Neurol. Sci. 160, 47-53.

Davis, G. W., Schuster, C. M., and Goodman, C. S. (1996). Genetic dissection of structural and functional components of synaptic plasticity. III. CREB is necessary for presynaptic functional plasticity. Neuron 17 , 669-679.

de Koning, D. J., and Haley, C. S. (2005). Genetical genomics in humans and model organisms. Trends Genet. 21, 377-381.

Empson, R. M., Garside, M. L., and Knopfel, T. (2007). Plasma membrane $\mathrm{Ca}^{2+}$ ATPase 2 contributes 
to short-term synapse plasticity at the parallel fiber to Purkinje neuron synapse. J. Neurosci. 27, 3753-3758.

Evans, M. L., Busselberg, D., and Carpenter, D. O. (1991). $\mathrm{Pb}^{2+}$ blocks calcium currents of cultured dorsal root ganglion cells. Neurosci. Lett. 129, 103-106.

Faroon, O., Jones, D., and de Rosa, C. (2000). Effects of polychlorinated biphenyls on the nervous system. Toxicol. Ind. Health 16, 305-333.

He, T., Hirsch, H. V. B., Ruden, D. M., and Lnenicka, G. A. (2009). Chronic lead exposure alters presynaptic calcium regulation and synaptic facilitation in Drosophila larvae. Neurotoxicology 30, 777-784.

Hirsch, H. V., Mercer, J., Sambaziotis, H., Huber, M., Stark, D. T., Torno-Morley, T., Hollocher, K., Ghiradella, H., and Ruden, D. M. (2003). Behavioral effects of chronic exposure to low levels of lead in Drosophila melanogaster. Neurotoxicology 24, 435-442.

Hirsch, H. V. B., Possidente, D., Averill, S., Despain, T. P., Buytkins, J., Thomas, V., Goebel, W. P., Shipp-Hilts, A., Wilson, D., Hollocher, K., Possidente, B., Lnenicka, G., and Ruden, D. M. (2009). Variations at a quantitative trait locus (QTL) affect development of behavior in lead-exposed Drosophila melanogaster. Neurotoxicology 30, 305-311.

Hovatta, I., Zapala, M. A., Broide, R. S., Schadt, E. E., Libiger, O., Schork, N. J., Lockhart, D. J., and Barlow, C. (2007). DNA variation and brain region-specific expression profiles exhibit different relationships between inbred mouse strains: implications for eQTL mapping studies. Genome Biol. 8, R25.

Jansen, R. C., and Nap, J. P. (2001). Genetical genomics: the added value from segregation. Trends Genet. 17, 388-391.

Jin, M., Guan, C.-B., Jiang, Y.-A., Chen, G., Zhao, C.-T., Cui, K., Song, Y.Q., Wu, C.-P., Poo, M.-M., and Yuan, X.-B. (2005). $\mathrm{Ca}^{2+}$-dependent regulation of rho GTPases triggers turning of nerve growth cones. J. Neurosci. 25, 2338-2347.

Keshishian, H., Broadie, K., Chiba, A., and Bate, M. (1996). The Drosophila neuromuscular junction: a model system for studying synaptic development and function. Annu. Rev. Neurosci. 19, 545-575.

Kiraly, E., and Jones, D. G. (1982). Dendritic spine changes in rat hippocampal pyramidal cells after postnatal lead treatment: a Golgi study. Exp. Neurol. 77, 236-239.

Levi, F., Okyar, A., Dulong, S., Innominato, P. F., and Clairambault, J. (2010). Circadian timing in cancer treatments. Annu. Rev. Pharmacol. Toxicol. 50, 377-421.

Li, J., and Burmeister, M. (2005). Genetical genomics: combining genetics with gene expression analysis. Hum. Mol. Genet. 14, R163-R169.

Li, X. M., Delaunay, F., Dulong, S., Claustrat, B., Zampera, S., Fujii, Y., Teboul, M., Beau, J., and Lévi, F. (2010). Cancer inhibition through circadian reprogramming of tumor transcriptome with meal timing. Cancer Res. 70, 3351-3360.

Li, Y., Álvarez, O. A., Gutteling, E. W., Tijsterman, M., Fu, J., Riksen, J. A. G., Hazendonk, E., Prins, P., Plasterk, R. H. A., Jansen, R. C., Breitling, R., and Kammenga, J. E. (2006). Mapping determinants of gene expression plasticity by genetical genomics in $C$. elegans. PLoS Genet. 2, e222. doi: 10.1371/journal.pgen.0020222

Li, Y., Breitling, R., and Jansen, R. C. (2008). Generalizing genetical genomics: getting added value from environmental perturbation. Trends Genet. 24, 518-524.

Lohmann, C., and Bonhoeffer, T. (2008). A role for local calcium signaling in rapid synaptic partner selection by dendritic filopodia. Neuron 59, 253-260.

MacDonald, J. F., Jackson, M. F., and Beazely, M. A. (2006). Hippocampal long-term synaptic plasticity and signal amplification of NMDA receptors. Crit. Rev. Neurobiol. 18, 71-84.

Mackay, T. F. C., and Anholt, R. R. H. (2006). Of flies and man: Drosophila as a model for human complex traits. Annu. Rev. Genomics Hum. Genet. 7, 339-367.

Majewski, J., and Pastinen, T. (2011). The study of eQTL variations by RNA-seq: from SNPs to phenotypes. Trends Genet. 27, 72-79.

Mas-Oliva, J. (1989). Effect of lead on the erythrocyte $\left(\mathrm{Ca}^{2+}, \mathrm{Mg}^{2+}\right)$ ATPase activity. Calmodulin involvement. Mol. Cell. Biochem. 89, 87-93.

Mayersbach, H. (1976). Time - a key in experimental and practical medicine. Arch. Toxicol. 36, 185-216.

Morley, E. J., Hirsch, H. V., Hollocher, K., and Lnenicka, G. A. (2003). Effects of chronic lead exposure on the neuromuscular junction in Drosophila larvae. Neurotoxicology 24, 35-41.

Mueller, M., Goel, A., Thimma, M., Dickens, N. J., Aitman, T. J., and Mangion, J. (2006). eQTL
Explorer: integrated mining of combined genetic linkage and expression experiments. Bioinformatics 22, 509-511.

Petit, T. L., and LeBoutillier, J. C. (1979). Effects of lead exposure during development on neocortical dendritic and synaptic structure. Exp. Neurol. 64, 482-492.

Prakash, S. (2011). Human metabolic individuality in biomedical and pharmaceutical research. Circ. Cardiovasc. Genet. 4, 714-715.

Pratt, K. G., Watt, A. J., Griffith, L. C., Nelson, S. B., and Turrigiano, G. G. (2003). Activity-dependent remodeling of presynaptic inputs by postsynaptic expression of activated CaMKII. Neuron 39, 269-281.

Rai, A., Maurya, S. K., Khare, P., Srivastava, A., and Bandyopadhyay, S. (2010). Characterization of developmental neurotoxicity of As, Cd, and $\mathrm{Pb}$ mixture: synergistic action of metal mixture in glial and neuronal functions. Toxicol. Sci. 118, 586-601.

Rockman, M. V., and Kruglyak, L. (2006). Genetics of global gene expression. Nat. Rev. Genet. 7, 862-872.

Rosato, E., and Kyriacou, C. P. (2006). Analysis of locomotor activity rhythms in Drosophila. Nat. Protoc. 1, 559-568.

Rosato, E., Tauber, E., and Kyriacou, C. P. (2006). Molecular genetics of the fruit-fly circadian clock. Eur. J. Hum. Genet. 14, 729-738.

Ruden, D. M., Chen, L., Possidente, D., Possidente, B., Rasouli, P., Wang, L., Lu, X., Garfinkel, M. D., Hirsch, H. V., and Page, G. P. (2009). Genetical toxicogenomics in Drosophila identifies master-modulatory loci that are regulated by developmental exposure to lead. Neurotoxicology 30, 898-914. Sandhir, R., and Gill, K. D. (1994a) Lead perturbs calmodulin dependent cyclic AMP metabolism in rat central nervous system. Biochem. Mol. Biol. Int. 33, 729-742.

Sandhir, R., and Gill, K. D. (1994b) Alterations in calcium homeostasis on lead exposure in rat synaptosomes. Mol. Cell. Biochem. 131, 25-33.

Shaw, P. J., Cirelli, C., Greenspan, R. J., and Tononi, G. (2000). Correlates of sleep and waking in Drosophila melanogaster. Science 287, 1834-1837.

Singh, M. P., Ram, K. R., Mishra, M., Shrivastava, M., Saxena, D. K., and Chowdhuri, D. K. (2010). Effects of co-exposure of benzene, toluene and xylene to Drosophila melanogaster: alteration in hsp70, hsp60, hsp83, hsp26, ROS generation and oxidative stress markers. Chemosphere 79 577-587.

Smith, E. N., and Kruglyak, L. (2008). Gene-environment interaction in yeast gene expression. PLoS Biol. 6, e83. doi: 10.1371/journal.pbio. 0060083

Suhre, K., Shin, S.-Y., Petersen, A.-K., Mohney, R. P., Meredith, D., Wägele, B., A.-K., Altmaier, E., CARDIoGRAM, Deloukas, P., Erdmann, J., Grundberg, E., Hammond, C. J., Hrabé de Angelis, M., Kastenmüller, G., Köttgen, A., Kronenberg, F., Mangino, M., Meisinger, C., Meitinger, T., Mewes, H.-W., Milburn, M. V., Prehn, C., Raffler, J., Ried, J. S., Römisch-Margl, W., Samani, N. J., Small, K. S., Wichmann, H.-E., Zhai, G., Illig, T., Spector, T. D., Adamski, J., Soranzo, N., and Gieger, C. (2011). Human metabolic individuality in biomedical and pharmaceutical research. Nature 477, 54-60.

Takahashi, J. S., Shimomura, K., and Kumar, V. (2008). Searching for genes underlying behavior: lessons from circadian rhythms. Science 322, 909-912.

UNEP. (2010). Lead Exposure and Human Health. Nairobi: Chemicals Branch, Division of Technology, Industry and Economics, United Nations Environment Program. Available at: www.chem.unep.ch/ pops/pdf/lead/leadexp.pdf [accessed January 3, 2012].

von Mayersbach, H. (1975). Proceedings: Time - a key in experimental and practical medicine. Naunyn Schmiedebergs Arch. Pharmacol. 287(Suppl.), R107.

West, M. A., Kim, K., Kliebenstein, D. J., van Leeuwen, H., Michelmore, R. W., Doerge, R. W., and St. Clair, D. A. (2007). Global eQTL mapping reveals the complex genetic architecture of transcript-level variation in Arabidopsis. Genetics 175 1441-1450.

$\mathrm{Xu}, \quad$ F., Hennessy, D. A., Lee, T. K. M., and Syed, N. I. (2009). Trophic factor-induced intracellular calcium oscillations are required for the expression of postsynaptic acetylcholine receptors during synapse formation between Lymnaea neurons. J. Neurosci. 29, 2167-2176.

Zhang, X., Huang, S., Sun, W. and Wang, W. (2012). Rapid and robust resampling-based multiple testing correction with application in genome-wide eQTL study. Genetics. doi: 10.1534/genetics.111.137737

Zucker, R. S. (1996). Exocytosis: a molecular and physiological perspective. Neuron 17, 1049-1055. 
Zucker, R. S., and Regehr, W. G. (2002). Short-term synaptic plasticity. Annu. Rev. Physiol. 64, 355-405.

Conflict of Interest Statement: The authors declare that the research was conducted in the absence of any commercial or financial relationships that could be construed as a potential conflict of interest

Received: 29 February 2012; paper pending published: 19 March 2012; accepted: 09 April 2012; published online: 04 May 2012.

Citation: Hirsch HVB, Lnenicka G, Possidente D, Possidente B, Garfinkel
MD, Wang $L, L u \quad X$ and Ruden, DM. (2012) Drosophila melanogaster as a model for lead neurotoxicology and toxicogenomics research. Front. Gene. 3:68. doi: 10.3389/fgene.2012. 00068

This article was submitted to Frontiers in Toxicogenomics, a specialty of Frontiers in Genetics.
Copyright (C) 2012 Hirsch, Lnenicka, Pos sidente, Possidente, Garfinkel, Wang, $L u$ and Ruden. This is an open-access article distributed under the terms of the Creative Commons Attribution Non Commercial License, which permits noncommercial use, distribution, and reproduction in other forums, provided the original authors and source are credited. 